\title{
The spin structure of the proton in a non-relativistic quark model
}

\author{
J. Keppler \\ Institut für theoretische Physik III, Universität Erlangen-Nürnberg, \\ Staudtstraße 7, 91058 Erlangen, Germany \\ H. M. Hofmann \\ Institut für theoretische Physik III, Universität Erlangen-Nürnberg, \\ Staudtstraße 7, 91058 Erlangen, Germany \\ and \\ European Center for Theoretical Studies in Nuclear Physics and Related Areas (ECT*), \\ Villa Tambosi, Via delle Tabarelle 286, I-38050 Villazzano (Trento), Italy
}

(September 30, 2018)

\begin{abstract}
The spin structure of the proton is investigated in the framework of an extended quark potential model which in addition to the conventional $3 q-$ structure also takes into account $(3 q)(q \bar{q})$-admixtures in the proton wave function. For reasons of parity such admixtures contain an odd orbital angular momentum thus allowing the proton spin to be shared among quark spins and orbital angular momenta. We show that only certain admixtures are suited for a significant reduction of the quark spin content of the proton as suggested by the EMC-result. Within a Hamiltonian model quark spin contributions to the proton spin down to 0.5 can be reproduced easily .
\end{abstract}

Typeset using REVTEX 
In 1988 the European Muon Collaboration (EMC) [4,2] determined the spindependent proton structure function $g_{1}^{p}(x)$ down to values of $x$ as small as 0.015. Furthermore an estimate for the extrapolation to $x \rightarrow 0$ was presented, thus being able to give a first result for the integral of $g_{1}^{p}(x)$. An evaluation of the underlying deep inelastic scattering process in the framework of the naive quark parton model results in associating the quantity $\int_{0}^{1} d x g_{1}^{p}(x)$ with a linear combination of the quark spin contributions to the proton spin. Applying additional information from neutron and hyperon $\beta$-decay it is then possible to analyze the spin structure of the proton. Such a straightforward analysis leads, however, to the surprising conclusion that the contribution of all the quarks to the nucleon spin is unexpectedly small and compatible with zero [3:4]. Accordingly a huge fraction of the proton spin has to be carried by gluonic degrees of freedom or internal orbital angular momenta.

The consequence for the nonrelativistic quark model and a possible way out of the "spin crisis" was first discussed by Jaffe and Lipkin [5] who modified the proton wave function in admixing $(3 q)(q \bar{q})$-components to the conventional $3 q-$ object. This can be expressed as

$$
\left|\Psi_{p}^{\mathrm{tot}}\right\rangle=\nu_{0}\left|\Psi_{p}(3 q)\right\rangle+\sum_{i=1} \nu_{i}\left|\Psi_{i}[(3 q)(q \bar{q})]\right\rangle .
$$

The structure of such $(3 q)(q \bar{q})$-components which can be considered as baryon-mesoncombinations is depicted in Fig. [1. Jaffe and Lipkin [5] took into account just two $5 q-$ components in which the $3 q$-substructure was assumed to have the quantum numbers of the proton and the odd orbital angular momentum which has to be introduced for reasons of parity was used to construct $\mathrm{P}$-wave vector mesons, one of them being coupled to $J_{M}=0$, the other to $J_{M}=1$. They found that the free coefficients $\nu_{1,2}$ of the total wave function can be chosen in a way to reproduce the weak coupling constants as well as a quark spin content which is compatible with zero. In such a scenario the proton spin is effectively carried by the orbital angular momentum $L_{M}$, see Fig. 1, which in a non-relativistic quark model is apart from the quark spins the only source of the nucleon spin.

A closer look at the Jaffe-Lipkin-ansatz [5], however, reveals several problems. First the choice of the proton wave function seems to be quite arbitrary and cannot be justified 
by physical arguments. The $q \bar{q}$-pairs taken into account are $\mathrm{P}$-wave vector mesons while on energetic grounds one would expect the $5 q$-component to be dominated by pseudoscalar S-wave mesons, like the pion. Second the fit of the coefficients leads to a complete suppression of the $3 q$-structure $\left|\Psi_{p}(3 q)\right\rangle$ and a dominance of the $5 q$-admixtures, a scenario which seems rather questionable. Finally the model does not specify the interaction generating the $q \bar{q}-$ pairs which may be regarded as effective sea quarks. This also prevents studying the behaviour of other important properties of the nucleon like mass and magnetic moment.

Nevertheless the simple model proposed by Jaffe and Lipkin [5] serves as a guideline to perform an investigation of the spin structure of the proton in an extended quark potential model [6.7]. In this model the interactions of the quarks are mediated by one gluon exchange potentials which because of the sea quark admixtures also include an annihilation as well as pair creation and pair destruction potentials. Together with the operators for kinetic energy and confinement the sum of these potentials constitutes the Hamiltonian for the proton state in the extended non-relativistic quark model. For details of the interaction see reference [7]. The free parameters of this Hamiltonian are the quark masses $m_{u}=m_{d}$ and $m_{s}$, the strong coupling constant $\alpha_{s}$, the confinement strength $a$, and a cutoff parameter $\Lambda$ which enters a form factor [9] of the quark-gluon-vertex. The latter is introduced in order to regularize the potential and make variational calculations possible. The approximate solution of the Schrödinger equation is then given by the Refined Resonating Group Method [8]. As an ansatz for the wave function we start from Eq. (1) allowing $5 q$-components which contain either $\mathrm{S}$ - or $\mathrm{P}$-wave mesons and properly antisymmetrize the quarks of the total wave function. In the case of S-wave mesons the odd orbital angular momentum is on the relative motion between the baryon and the meson,i.e. $L_{\mathrm{rel}}=1$, in the other case $L_{\text {rel }}=0$ and $L_{M}=1$. Admixtures with more than five particles, orbital angular momenta of three, or more complex angular momentum configurations are energetically suppressed and therefore neglected. Altogether 66 baryon-meson-combinations which can be coupled to the quantum numbers of the proton are taken into account, among them also ones containing strange quarks and color octet fragments. They are called channels and determined by the 
following coupling scheme in spin, isospin or color space, see Fig. 1 for the obvious notation,

$$
\begin{aligned}
{\left[J_{p} T_{p} C_{p}\right]=} & {\left[\left[J_{B} \times\left[S_{M} \times L_{M}\right] J_{M}\right] S_{c} \times L_{\mathrm{rel}}\right] J_{p} } \\
& \otimes\left[T_{B} \times T_{M}\right] T_{p} \otimes\left[C_{B} \times C_{M}\right] C_{p}
\end{aligned}
$$

where $S_{c}$ is the channel spin. The coefficients $\nu_{i}$ of the individual channels are obtained by diagonalizing the Hamiltonian matrix and are therefore no free parameters in our model.

Before starting the numerical calculations we study the spin properties of individual channels. These investigations reveal that only certain classes of $5 q$-components meet the requirements for an appreciable reduction of the quark spin contribution. To keep the notation simple, we classify only those channels which will be discussed later on. For channels containing S-wave mesons it is sufficient to specify the channel spin $S_{c}$, therefore we denote the channels by $\left|S_{c} ; \mathrm{S}\right\rangle$. Of the channels containing $\mathrm{P}$-wave mesons only those play a special role where the baryon has the quantum number $J_{B}=1 / 2$, but the spin $S_{M}$ and the total angular momentum $J_{M}$ have to be specified, hence, we denote them by $\left|S_{M}, J_{M} ; \mathrm{P}\right\rangle$. On the one hand the quark spin contribution to the nucleon spin can be reduced by channels which have a negative total spin content of the quarks. Into this class, in the following designated as class-I, fall all channels with $|1 / 2 ; \mathrm{S}\rangle$ and $|0,1 ; \mathrm{P}\rangle$. Examples for the former and latter category are $\mathrm{N} \pi, \mathrm{N} \rho, \mathrm{N} \omega, \mathrm{N} \eta, \Delta \rho$, and $\mathrm{Nb}_{1}(1235), \mathrm{Nh}_{1}(1170)$ [1] respectively. It is straightforward to show that

$$
\begin{aligned}
& \left\langle 1 / 2 ; \mathrm{S}\left|\sigma_{z}\right| 1 / 2 ; \mathrm{S}\right\rangle \\
= & \left\langle 0,1 ; \mathrm{P}\left|\sigma_{z}\right| 0,1 ; \mathrm{P}\right\rangle=-\frac{1}{3},
\end{aligned}
$$

holds. Since different channels of this class do not mix via the spin operator, the admixture of such $5 q$-structures to the conventional $3 q$-structure will therefore in any case result in a reduction of the quark spin content $\Sigma\left(\Psi_{p}^{\text {tot }}\right) \equiv\left\langle\Psi_{p}^{\text {tot }}\left|\sigma_{z}\right| \Psi_{p}^{\text {tot }}\right\rangle$ of the physical proton. All remaining channels are characterized by a positive quark spin contribution. The second possibility to reduce $\Sigma\left(\Psi_{p}^{\text {tot }}\right)$ then consists in admixing channels which are coupled strongly via the spin operator. Into this class, in the following designated as class-II, fall channels 
containing $\mathrm{P}$-wave vector mesons differing by the $J_{M}$-quantum number only. Examples for such pairs are $\left(\mathrm{Na}_{0}(980), \mathrm{Na}_{1}(1260)\right)$ and $\left(\mathrm{Nf}_{0}(975), \mathrm{Nf}_{1}(1285)\right)$ [11]. A simple calculation yields

$$
\begin{aligned}
& \left\langle 1,0 ; \mathrm{P}\left|\sigma_{z}\right| 1,0 ; \mathrm{P}\right\rangle=1 \\
& \left\langle 1,1 ; \mathrm{P}\left|\sigma_{z}\right| 1,1 ; \mathrm{P}\right\rangle=\frac{1}{3} \\
& \left\langle 1,0 ; \mathrm{P}\left|\sigma_{z}\right| 1,1 ; \mathrm{P}\right\rangle=-\frac{2 \sqrt{2}}{3} .
\end{aligned}
$$

Incorporating one of these channel doublets into the ansatz of their wave function, Jaffe and Lipkin [5] took a very suitable choice and succeeded in fitting the coefficients to reproduce the $\beta$-dacay as well as the EMC-data. Performing model calculations based on a Hamiltonian to determine the nucleon wave function, it is quite evident, however, that the corresponding expansion coefficients of these channel pairs are no fit parameters and therefore play a crucial role. If they are too small or have the wrong sign no reduction of the total quark spin content can be expected.

Having analyzed the spin properties of individual channels, we proceed to investigate the influence of several relevant channels on the spin structure of the proton. As a first step we determine the maximal strength of the $3 q$-structure in the nucleon wave function which does not contradict the experimental finding for the quark spin content. Admixing an arbitrary number of channels out of the $|1 / 2 ; \mathrm{S}\rangle$ - or $|0,1 ; \mathrm{P}\rangle$-sectors, one simply gets

$$
\Sigma\left(\Psi_{p}^{\mathrm{tot}}\right)=\nu_{0}^{2}-\frac{1}{3} \sum_{i=1} \nu_{i}^{2}=\frac{4}{3} \nu_{0}^{2}-\frac{1}{3},
$$

using $\sum_{i=0} \nu_{i}^{2}=1$. Thus, in this case the quark spin content depends on the probability amplitude of the $3 q$-component only. According to the previous paragraph a second promising ansatz consists in including channel doublets with a large negative spin overlap, those with a positive one do not exist. This results in a more complex expression for $\Sigma\left(\Psi_{p}^{\text {tot }}\right)$ which in addition to $\nu_{0}$ also involves the coefficients of the $5 q$-components. From such expressions we altogether find that even after restricting to the most favourable channel combinations and an optimal choice of the various coefficients a value of $\Sigma\left(\Psi_{p}^{\text {tot }}\right) \approx 0$ can be achieved only if 
$\nu_{0}^{2} \leq 0.3$. Taking into account the possibility of $\Sigma\left(\Psi_{p}^{\text {tot }}\right) \approx 0.5$, the squared amplitude of the $3 q$-structure has to be less than $\approx 0.7$.

After having studied the fundamental connection between the basic channels and the quark spin content of the proton at an analytic level it is now of central interest which coefficients and which spin structure are realized in the extended quark potential model. As an appropriate starting point we employ a previously given parameter set [10], with $m_{u}=$ $m_{d}=330 \mathrm{MeV}, \alpha_{s}=1.7, a=-19 \mathrm{MeV} / \mathrm{fm}^{2}$, extended by $m_{s}=600 \mathrm{MeV}$ and $\Lambda=300 \mathrm{MeV}$. The calculations yield that solely the admixture of channels out of the $|1 / 2 ; \mathrm{S}\rangle-$ and $|0,1 ; \mathrm{P}\rangle-$ sectors leads to a reduction of the total quark spin content of the proton. In this case the probability amplitude of the $3 q$-structure is $\nu_{0}^{2} \approx 0.85$ which corresponds to $\Sigma\left(\Psi_{p}^{\text {tot }}\right) \approx 0.80$ according to eq. (7). It also appears that the essential contribution to this effect stems from the five most dominant class-I channels $\mathrm{N} \pi, \mathrm{N} \rho, \mathrm{N} \omega, \mathrm{N} \eta$, and $\Delta \rho$. These are therefore the relevant candidates for further considerations. Channel doublets with a large negative spin overlap, such as $\left(\mathrm{Na}_{0}, \mathrm{Na}_{1}\right)$ and $\left(\mathrm{Nf}_{0}, \mathrm{Nf}_{1}\right)$ out of class-2, couple comparatively well to the $3 q$-component. The value of $\Sigma\left(\Psi_{p}^{\text {tot }}\right)$, however, even increases with respect to the one of the $3 q$-proton. This means that the corresponding expansion coefficients resulting from our quark model calculations are not suited for decreasing the quark spin content at all.

Finally we would like to study whether and to which degree a further reduction of $\Sigma\left(\Psi_{p}^{\text {tot }}\right)$ is obtainable within the limits of an acceptable variation of the parameter set which also reproduces fundamental properties of the nucleon like mass $m_{p}$, magnetic moment $\mu_{p}$, and the $\beta$-decay constant $\left(g_{A} / g_{V}\right)_{\mathrm{np}}$ of the neutron. Since we restrict ourselves to the dominant $5 q$-structures and therefore omit channels with strange quarks, the parameters remaining for a variation are $m_{q} \equiv m_{u}=m_{d}, \alpha_{s}$, and $a$. The cutoff is kept fixed to the value of $\Lambda=300 \mathrm{MeV}$. We find in the case of class -2 channel admixtures parameter sets which both reproduce the basic properties of the nucleon and lead to a significant reduction of the $3 q$-amplitude in the wave function. This, however, does not lead to a decrease of the quark spin content because parameter variations do not change the unsuitable phases of the expansion coefficients. On the other hand class- 1 channel admixtures guarantee a 
reduction of the quark spin in any case. We obtain $\Sigma\left(\Psi_{p}^{\text {tot }}\right) \approx 0.5$ by fixing the parameters at $m_{q}=250 \mathrm{MeV}, \alpha_{s}=4.0$, and $a=-170 \mathrm{MeV} / \mathrm{fm}^{2}$. These values lie within the range of already existing parameter sets in the literature [10, 12, 13]. We therefore believe that a parameter set leading to a proton configuration in which quark spins and orbital angular momenta share the nucleon spin equally is quite reasonable. It remains to be mentioned that our calculation yields in addition to the proton mass, the magnetic moment of the nucleon, and the $\mathrm{N}-\Delta$-mass splitting also a $\beta$-decay constant $\left(g_{A} / g_{V}\right)_{\mathrm{np}}=1.23$ which is in perfect agreement with experiment. This means that the extended quark potential model cures a well known shortcoming of the conventional non-relativistic quark model, namely the overestimated ratio of the weak coupling constants. Any further sizable reduction of the quark spin content in the nucleon, however, would require an enormous modification of the original parameter set which by no means seems to be acceptable to us. Moreover, in this case the agreement between the calculated and the experimentally determined static properties of the nucleon deteriorates.

In summary we have performed a careful analysis of individual $5 q$-channels with respect to their angular momentum structure. For reasons of parity these channels contain an odd orbital angular momentum thus allowing the proton spin to be shared among quark spins and orbital angular momenta. We demonstrated that only certain baryon-meson-combinations are in principle suited for a reduction of the quark spin contribution to the nucleon spin. These are either channels with a negative quark spin content or channel doublets with a huge negative spin overlap. In the framework of consistent quark model calculations we found, however, that an admixture of such channel doublets does not lead to a reduction of the quark spin in the nucleon. The success of the Jaffe-Lipkin-fit [5] is therefore not reproducable in an extended quark potential model. Choosing on the other hand a wave function which in addition to the $3 q$-structure consists of the channels $\mathrm{N} \pi, \mathrm{N} \rho, \mathrm{N} \omega, \mathrm{N} \eta$ and $\Delta \rho$, one can reproduce quark spin contributions down to $50 \%$. This result is the lower bound which can be achieved employing a reasonable parameter set and clearly demonstrates the limitations of non-relativistic quark models if the EMC-result should be confirmed in the 
future.

We would like to acknowledge helpful discussions with M. Unkelbach and H. Berger. 


\section{REFERENCES}

[1] J. Ashman et al., Phys. Lett. B206, 364 (1988)

[2] J. Ashman et al., Nucl. Phys. B328, 1 (1989)

[3] S. J. Brodsky, J. Ellis and M. Karliner, Phys. Lett. B206, 309 (1988)

[4] H. J. Lipkin, Phys. Lett. B256, 284 (1991)

[5] R. L. Jaffe and H. J. Lipkin, Phys. Lett. B266, 458 (1991)

[6] Y. Fujiwara and K. T. Hecht, Nucl. Phys. A444, 541 (1985)

[7] R. Müller and H. M. Hofmann, Phys. Rev. Lett. 65, 3245 (1990)

[8] H. M. Hofmann, in Models and Methods in Few Body Physics, edited by L. S. Ferreira, Lecture Notes in Physics 273, 243 (Springer, Berlin, 1987)

[9] R. Machleidt, K. Holinde and C. Elster, Phys. Rep. 149, 1 (1987)

[10] O. Morimatsu, M. Oka and K. Yazaki, Nucl. Phys. A424, 412 (1984)

[11] M. Aguilar-Benitez et al. (Particle Data Group), Phys. Rev. D45, No.11, Part II (June 1992)

[12] S. Godfrey and N. Isgur, Phys. Rev. D32, 189 (1985)

[13] Y. Fujiwara and K. T. Hecht, Nucl. Phys. A463, 255c (1987) 


\section{FIGURES}

FIG. 1. The structure of $(3 q)(q \bar{q})$-admixtures in angular momentum space. 
This figure "fig1-1.png" is available in "png" format from: http://arxiv.org/ps/hep-ph/9407258v1 